\title{
The Relationship between Health Literacy and Health Behaviour in People with Diabetes: A Danish Population-Based Study
}

\author{
Karina Friis, ${ }^{1}$ Benedicte Donslund Vind, ${ }^{1}$ \\ Rebecca K. Simmons, ${ }^{2,3,4}$ and Helle Terkildsen Maindal ${ }^{5,6}$ \\ ${ }^{1}$ DEFACTUM, Public Health \& Health Services Research, Central Denmark Region, Olof Palmes Allé 15, 8200 Aarhus N, Denmark \\ ${ }^{2}$ Department of Public Health, Section of General Practice, Aarhus University, Aarhus, Denmark \\ ${ }^{3}$ Danish Diabetes Academy, Odense, Denmark \\ ${ }^{4}$ Medical Research Council Epidemiology Unit, University of Cambridge School of Clinical Medicine, Cambridge, UK \\ ${ }^{5}$ Steno Diabetes Center, SCHP, Niels Steensens Vej 6, Gentofte, Denmark \\ ${ }^{6}$ Department of Public Health, Aarhus University, Bartholins Allé 2, 8000 Aarhus C, Denmark
}

Correspondence should be addressed to Helle Terkildsen Maindal; htm@ph.au.dk

Received 16 April 2016; Accepted 6 September 2016

Academic Editor: Diane Levin-Zamir

Copyright (C) 2016 Karina Friis et al. This is an open access article distributed under the Creative Commons Attribution License, which permits unrestricted use, distribution, and reproduction in any medium, provided the original work is properly cited.

Background. People with diabetes who have poor health behaviours are at greater risk for a range of adverse health outcomes. We aimed to investigate the relationship between health literacy and health behaviour (smoking, alcohol, physical activity, and diet) in people with diabetes. Methods. The study was based on respondents aged 25 years or older from a population-based survey in 2013 who reported having diabetes $(n=1685)$. Two dimensions from the Health Literacy Questionnaire were used: "understand health information" and "actively engage with healthcare providers." We used logistic regression to examine the association between health literacy and health behaviour. Results. After adjustment for sociodemographic factors, individuals with diabetes who found it difficult to understand information about health had higher odds of being physically inactive (OR: $3.43,95 \%$ CI: 2.14-5.51) and having unhealthy dietary habits (OR: 3.01, 95\% CI: 1.63-5.58). Similar results were observed for individuals who found it difficult to actively engage with healthcare providers. No associations were found between the two dimensions of health literacy and smoking and alcohol consumption. Conclusion. When developing health services and interventions to improve health behaviour among people with diabetes, our results suggest that they may benefit by including focus on health literacy.

\section{Background}

People with diabetes are at risk for a range of adverse health outcomes, including heart attacks, strokes, amputations, blindness, and end-stage renal disease [1]. Many of these adverse health outcomes can be prevented or delayed if people with diabetes maintain a healthy lifestyle in relation to diet, physical activity, alcohol, and smoking [2,3]. Thus, it is important for health services and healthcare providers to develop strategies and interventions to help people with diabetes to improve and maintain their health behaviour.

Health literacy is defined as the cognitive and social skills that determine a person's motivation and ability to gain access to, understand, and use information in ways that promote and maintain good health [4]. Health literacy brings together many concepts that relate to what people need in order to make effective decisions about health for themselves and their families. A recent Danish population-based study has shown that, even after adjusting for sociodemographic factors and comorbidities, people with diabetes find it more difficult to understand health information than the general population [5].

Diabetes is a chronic disease characterized by a high level of complexity that requires extensive self-care management including translation of guidelines into everyday life [6]. The demands for people with diabetes are complicated because self-care of a chronic disease often relies on information in printed educational materials, verbal instructions, and patient education courses [7]. People with low levels of health literacy may struggle to find and follow these instructions, 
when they are to be integrated in everyday life. Furthermore, low levels of health literacy are associated with poor glycaemic control in diabetes patients [8], as well as with a number of diabetic complications [9]. Therefore, having adequate health literacy is critical for diabetes patients for managing their condition and navigating the healthcare environment.

Some studies have shown that inadequate health literacy is associated with unhealthy behaviours such as smoking, physical inactivity, and poor diet in the general population [10-14], while one study [15] shows that health literary is not independently associated with some health behaviours. Few studies have investigated the relationship between health literacy and health behaviour such as smoking, alcohol consumption, physical activity, and diet in people with diabetes [16-20]. These studies showed no association between health literacy and these health behaviours. However, these studies were all conducted in small clinical settings and only measured health literacy in terms of cognitive and functional skills such as reading ability. To the best of our knowledge, no studies have investigated the association between health literacy and health behaviour in people with diabetes using a more comprehensive measure of health literacy including social and communication skills.

Using data from a large population-based survey, we aimed to investigate the association between health literacy and health behaviour (smoking, alcohol consumption, physical activity, and diet) in people with diabetes.

\section{Methods}

2.1. Study Design and Data Collection. The study is based on data from respondents aged 25 years or older from the 2013 Danish health and morbidity survey called "How Are You?" Geographically, Denmark is divided into five administrative regions and this study comprises data from one of these regions, the Central Denmark Region, where approximately $22 \%$ of the Danish population lives. Regarding sociodemographic and health related factors, the population of the Central Denmark Region is similar to the whole Danish population [21].

The survey consisted of a random sample of 46,354 people who were drawn from the Danish Civil Registration System. People were invited to complete a postal or a web-based questionnaire. Three reminders were issued. Data were collected by the Central Denmark Region between February and April 2013. In total, 29,473 people (63.6\%) completed and returned the questionnaire. The questionnaire included an item on diabetes status; 1,685 individuals $(5.7 \%)$ reported having diabetes.

\subsection{Measures}

2.2.1. Health Literacy. The Health Literacy Questionnaire (HLQ) is a widely used measure of health literacy that has been translated into many European and Asian languages [22]. The HLQ consists of nine dimensions and was developed using a validity-driven approach including in-depth grounded consultations, psychometric analyses, and cognitive interviews [22]. The translation and cultural adaption of the questions from English into Danish followed a rigorous forward-backward translation procedure and cognitive testing to ensure cross-cultural validity [23].

In the health and morbidity survey, two of the nine HLQ dimensions were included: "understand health information well enough to know what to do" to measure the functional dimension and "actively engage with healthcare providers" to measure the communicative dimension. Given that population surveys have limited space for survey questions, only these two scales were selected from the HLQ. The two scales cover two distinct elements of health literacy which we hypothesized would provide valuable insight into the health literacy challenges of individuals with chronic diseases. Each scale comprised five items where participants indicated their response on a four-point scale: $1=$ very difficult, $2=$ difficult, $3=$ easy, and $4=$ very easy. Scale scores were calculated as the mean of the five-item scores and then standardized to range between 1 (lowest ability) and 4 (highest ability) to ensure consistency with the response options. If responses to more than two items on a scale were missing for an individual, the scale score for that individual was regarded as missing. As a result of this, 137 observations (7.5\%) were excluded from the "understand health information" scale and 131 observations $(7.2 \%)$ from the "actively engage with healthcare providers" scale. Cronbach's alpha coefficients indicated that the internal consistency of both scales was high: "understand health information" $\alpha=0.86$ and "actively engage with healthcare providers" $\alpha=0.90$. The scales correlated positively with one another (Pearson's coefficient $=0.78$ ). We dichotomised the scale to identify individuals who found it very difficult or difficult $($ score $\leq 2)$ to understand health information or to actively engage with healthcare providers.

2.2.2. Health Behaviour. Four measures of health behaviour (smoking, alcohol consumption, physical inactivity, and unhealthy diet) were used. Respondents who indicated that they smoked on a daily basis were classified as smokers. Respondents were asked how many alcoholic drinks per week they normally drink. High-risk alcohol consumption was categorized in accordance with the Danish Health Authority's recommendations, that is, more than 21 drinks weekly for men and 14 drinks for women. Respondents were classified as physically inactive if, during a typical week, they were not physically active at least one day for a minimum of 30 minutes. Dietary habits were assessed using the validated Diet Quality Score [24], which classifies diet quality in relation to cardiovascular risk. The scale consists of 25 items including questions about type of bread spread, fats used for cooking, and how often the participants consumed selected food items (including fish, meat, fruits, and vegetables). The diet score was calculated and categorized into two groups: unhealthy diet and very healthy/reasonably healthy diet. Unhealthy diet was defined as having low intake of fruit, vegetables, and fish and a high amount of saturated fat [24].

2.2.3. Demographic and Socioeconomic Factors. Data on age, gender, ethnic background, and marital status were collected from national registers to achieve complete data. Respondents were defined as Danish if they had Danish citizenship 
or if at least one of their parents was a Danish citizen. Marital status refers to whether an individual is married or not. Information about educational attainment was derived from survey data. The participants were asked about their highest level of completed school education and any further higherlevel education. We categorized educational attainment as low (1-10 years), medium (11-14 years), and high ( $\geq 15$ years).

2.3. Ethics. The study was approved by the Danish Data Protection Agency and was conducted in accordance with the Helsinki Declaration. Information about the survey was provided to potential participants in writing and via the web. The participants' voluntary completion and return of the survey questionnaires constituted implied consent.

2.4. Statistical Analysis. The unique personal identification number given to all Danish citizens was used to link both respondents and nonrespondents to Danish national registers. A weight was constructed using a model-based calibration approach based on register information from Statistics Denmark. The weight accounted for differences in selection probabilities and response rates between subgroups. Data was weighted to represent the population of the Central Denmark Region and was used in all the data analyses.

To examine the association between health literacy and health behaviour in people with diabetes, eight logistic regression models were conducted, one for each health literacy dimension with the four different health behaviour measures (daily smoking, high-risk alcohol consumption, physical inactivity, and unhealthy diet) as dependent variables. In each logistic regression model, the odds ratios were further adjusted for gender, age, ethnic background, educational attainment, and marital status.

Significance was set at $p<0.05$. Statistical analyses were performed using STATA 13.

\section{Results}

Table 1 describes participant characteristics in relation to sociodemographic factors, the two health literacy dimensions, and health behaviour. Of the 1,685 individuals with diabetes, $34.1 \%$ had a low level of education. The majority of the participants were of Danish origin. In total, $9.3 \%$ of the participants found it difficult or very difficult to understand health information, and $9.3 \%$ found it difficult or very difficult to actively engage with healthcare providers. $11.8 \%$ of our sample had difficulties on at least one of the two scales (data not shown). The health behaviour characteristics of the participants show that $21.1 \%$ were daily smokers, $6.5 \%$ had high-risk alcohol consumption, $30.7 \%$ were physically inactive, and $12.3 \%$ had unhealthy dietary habits.

Nonresponse in the ten health literacy items was low and evenly distributed (between 5.3\% and 8.2\%) (Table 2), suggesting that the items were understood and had acceptable content. For all items, all response options were endorsed by some respondents although there were fewer in the extreme "very difficult" category and many in the "easy" category (Table 2).
TABLE 1: Characteristics of participants with diabetes from the "How Are You?” survey, Central Denmark Region (2013) $(N=1,685)$.

\begin{tabular}{|c|c|c|}
\hline & $N$ & $\%$ \\
\hline \multicolumn{3}{|l|}{ Sociodemographic factors } \\
\hline \multicolumn{3}{|l|}{ Gender } \\
\hline Male & 954 & 54.9 \\
\hline Female & 731 & 45.1 \\
\hline \multicolumn{3}{|l|}{ Age (years) } \\
\hline $25-44$ & 115 & 9.5 \\
\hline $45-64$ & 652 & 39.5 \\
\hline $65-84$ & 866 & 47.1 \\
\hline $85+$ & 52 & 4.0 \\
\hline \multicolumn{3}{|l|}{ Educational attainment } \\
\hline Low & 529 & 34.1 \\
\hline Medium & 790 & 48.9 \\
\hline High & 282 & 17.1 \\
\hline \multicolumn{3}{|l|}{ Ethnic background } \\
\hline Danish & 1633 & 95.0 \\
\hline Non-Danish & 52 & 5.0 \\
\hline \multicolumn{3}{|l|}{ Marital status } \\
\hline Living alone & 479 & 38.4 \\
\hline Married/cohabiting & 1170 & 61.6 \\
\hline \multicolumn{3}{|l|}{ Health literacy dimensions } \\
\hline \multicolumn{3}{|c|}{ Understand health information } \\
\hline Difficult/very difficult & 121 & 9.3 \\
\hline Easy/very easy & 1446 & 90.7 \\
\hline \multicolumn{3}{|c|}{ Mean scale score $(2.92$, SD 0.61$)$} \\
\hline \multicolumn{3}{|c|}{ Actively engage with healthcare providers } \\
\hline Difficult/very difficult & 133 & 9.3 \\
\hline Easy/very easy & 1438 & 90.7 \\
\hline \multicolumn{3}{|c|}{ Mean scale score $(3.00$, SD 0.62$)$} \\
\hline \multicolumn{3}{|l|}{ Health behaviours } \\
\hline \multicolumn{3}{|l|}{ Daily smoker } \\
\hline No & 1319 & 78.9 \\
\hline Yes & 330 & 21.1 \\
\hline \multicolumn{3}{|c|}{ High-risk alcohol consumption ${ }^{1}$} \\
\hline No & 1427 & 93.5 \\
\hline Yes & 102 & 6.5 \\
\hline \multicolumn{3}{|l|}{ Physically inactive $e^{2}$} \\
\hline No & 1172 & 69.3 \\
\hline Yes & 457 & 30.7 \\
\hline \multicolumn{3}{|l|}{ Unhealthy dietary habits ${ }^{3}$} \\
\hline No & 1390 & 87.7 \\
\hline Yes & 168 & 12.3 \\
\hline
\end{tabular}

${ }^{1} \geq 21$ drinks/week for men and $\geq 14$ drinks/week for women.

${ }^{2}$ Max. 30 minutes of physical activity one day during a typical week.

${ }^{3}$ Low intake of fruit, vegetables, and fish, and a high amount of saturated fat.

Table 3 describes the association between health literacy and health behaviour in people with diabetes. After adjusting for gender, age, ethnic background, educational affiliation, and cohabitation status, people who found it difficult to understand information about health had higher odds of 
TABLE 2: Response distribution and missing values for each item of the two health literacy scales.

\begin{tabular}{|c|c|c|c|c|c|}
\hline & $\begin{array}{c}\text { Very easy } \\
(\%)\end{array}$ & $\begin{array}{c}\text { Easy } \\
(\%)\end{array}$ & $\begin{array}{l}\text { Difficult } \\
(\%)\end{array}$ & $\begin{array}{c}\text { Very } \\
\text { difficult (\%) }\end{array}$ & $\begin{array}{c}\text { Item } \\
\text { missing (\%) }\end{array}$ \\
\hline \multicolumn{6}{|l|}{ Understanding health information well enough to know what to do } \\
\hline Confidently fill in medical forms in the correct way & 19.9 & 50.6 & 16.3 & 5.8 & 7.4 \\
\hline Accurately follow the instructions from healthcare providers & 17.0 & 51.8 & 20.2 & 2.9 & 8.2 \\
\hline Read and understand written health information & 19.1 & 53.6 & 15.6 & 4.7 & 6.8 \\
\hline Read and understand all the information on medication labels & 16.9 & 49.7 & 20.2 & 6.9 & 6.3 \\
\hline Understand what healthcare providers are asking you to do & 19.8 & 59.8 & 11.0 & 2.5 & 7.0 \\
\hline \multicolumn{6}{|l|}{ Ability to actively engage with healthcare providers } \\
\hline Make sure that healthcare providers understand your problems properly & 20.2 & 49.1 & 19.5 & 3.4 & 7.9 \\
\hline Feel able to discuss your health concerns with a healthcare provider & 23.3 & 53.8 & 14.6 & 2.0 & 6.4 \\
\hline Have good discussions about your health with doctors & 25.0 & 53.6 & 13.5 & 2.5 & 5.3 \\
\hline Discuss things with healthcare providers until you understand all you need to & 19.6 & 51.8 & 17.1 & 3.7 & 7.8 \\
\hline Ask healthcare providers questions to get the health information you need & 20.5 & 54.0 & 14.9 & 3.4 & 7.6 \\
\hline
\end{tabular}

being physically inactive (OR: 3.43, 95\% CI: 2.14-5.51) and having unhealthy dietary habits (OR: 3.01, 95\% CI: $1.63-$ 5.58). Similarly, people who found it difficult to actively engage with healthcare providers had higher odds of being physically inactive (OR: 2.72, 95\% CI: 1.76-4.20) and having unhealthy dietary habits (OR: 2.73, 95\% CI: 1.51-4.94). No significant results were found for the association between the two dimensions of health literacy and cigarette smoking and alcohol consumption.

\section{Discussion}

Results from this large population-based survey suggest that $9 \%$ of the participants found it difficult or very difficult to understand health information, and $9 \%$ found it difficult or very difficult to actively engage with healthcare providers. Respondents who found it difficult to understand information about health had higher odds of being physically inactive and having unhealthy dietary habits. Similar results were seen for people who found it difficult to actively engage with healthcare providers.

For diabetes patients with low health literacy levels, it may be difficult to navigate the large number of recommendations on diet and physical activity behaviour. These are complex behaviours that everyone uses on a daily basis and are subject to a number of individual and societal pressures that may be difficult to change. On the contrary, recommendations about smoking and alcohol consumption are generally more straightforward and have been promoted for several decades now. For example, the Danish Health Authority has run several antismoking campaigns and Denmark has continually undergone legislative changes with regard to smoking during the last decade, for example, tax on cigarettes and smoking bans at restaurants and public areas [25]. This attention to smoking may have led to high awareness about the risk of smoking in the Danish population and also among people with diabetes, and therefore information on smoking risk might be easier to understand, regardless of health literacy level compared with other health behaviours.
In contrast with our results, most research in individuals with diabetes does not support an association between health literacy and health behaviour such as physical activity and dietary habits [16-20]. However, research on health literacy in people with diabetes has focused on a one-dimensional concept of health literacy, that is, verbal ability. Furthermore, research has been conducted in clinical settings making it difficult directly to compare our results with other studies. For example, Bains and Egede showed no association between health literacy and physical activity and diet [16]. However, their study only included 125 adults recruited from a primary care clinic in the United States. Additionally, they assessed health literacy by asking patients to pronounce medical words, thus having a more narrow measure of health literacy than in our study. Kim et al. also found no association between health literacy and health behaviour, but they too had a small clinical sample consisting of 92 patients and the researchers only measured reading abilities [17]. In another study, smoking, physical activity, and diet were not significantly associated with health literacy [18]. These results on smoking are similar to our study. However, only 50 African Americans participated in the study, and health literacy was measured in terms of pronunciation and reading ability.

4.1. Implications. People with diabetes often have an ongoing interaction with the healthcare system and meet many healthcare practitioners throughout the life course. The challenges of adhering to public health recommendations concerning diet and physical activity are well known, particularly among patients with long-term conditions such as diabetes. Patients with long-term conditions such as diabetes need support to develop and maintain their health literacy skills. Our study suggests that it is difficult for patients with diabetes and low health literacy levels to adhere to recommended treatment guidelines. Adequate health literacy is crucial for patients to make optimal choices for their health. Healthcare providers therefore need to be aware of health literacy oriented strategies to support patients in making such choices. One strategy is to educate healthcare providers to communicate 


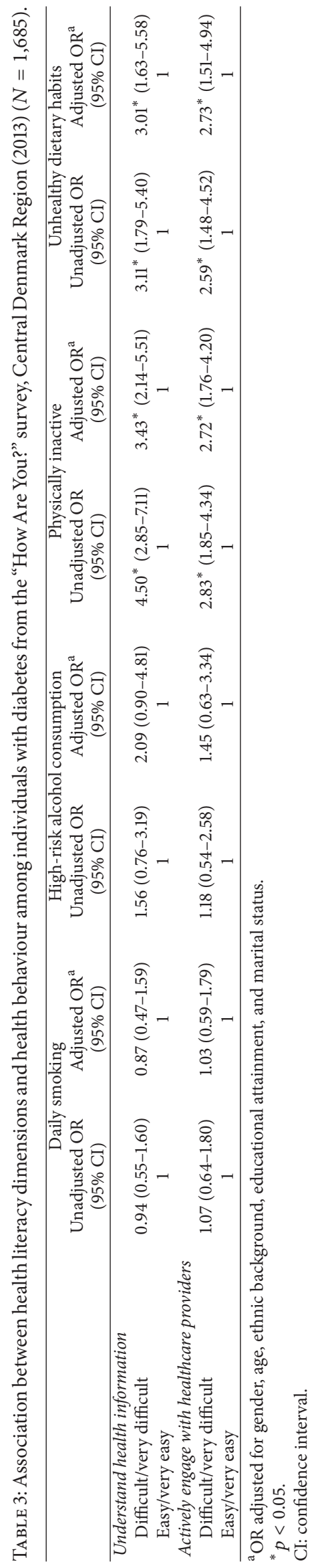


health information so that it is tailored to develop patients' understanding of their health condition and how to manage it. Exploring health literacy levels in more detail among individuals with diabetes with newly developed and validated tools is also a promising avenue of research [26].

4.2. Strengths and Limitations. We used data from a large population-based survey with a high response rate. As the survey was not focussed on individuals with diabetes, this may have lowered the risk of social desirability bias when responding to questions on health behaviour and health literacy level. An advantage of using a population-based sample for this study was that we included diabetes patients in the long maintenance phase of living with the disease. Many clinical studies only include individuals at the time of diagnosis or when adverse health outcomes cause them to use the healthcare services. The self-reported prevalence of diabetes was $5.7 \%$ in our study sample. This agrees well with data from the Danish National Diabetes Register [27], which shows that $6 \%$ of the Danish population above the age of 16 has diabetes. Another strength of this study was that we had the opportunity to control for a wide range of sociodemographic factors. Furthermore, to date, most health literacy research has focused on reading ability and numeracy based on data collected through direct testing procedures [28-30]. We used two different self-reported dimensions of health literacy that capture a dynamic state depending on how the individual person perceives his or her current situation.

Our findings are based on cross-sectional data, and therefore no conclusions about the temporality or causation can be made. Also, we were unable to differentiate between individuals with type 1 and type 2 diabetes. Health literacy and health behaviours might be different in these two groups. Also, it should be noted that there may be some imprecision and bias associated with using self-report measures of behaviour. Furthermore, the ability and motivation to fill out a health survey may be viewed as a health literacy competency in itself; thus, the most vulnerable groups may have been excluded from our study. As the questionnaire was not translated into other languages, people who had limited Danish language skills may not have participated in the survey. The study is also limited by including only two of the nine defined dimensions of the HLQ. Thus, it suffers from construct underrepresentation [31]. We can therefore draw conclusions only about the two dimensions we measured and not about health literacy overall. Application of the complete tool was not possible for practical reasons in this large population survey. We dichotomised the health literacy dimensions to be able to differentiate between respondents who found it "difficult" and "easy" to understand health information. This may have reduced the power to explore potential associations. However, using the exposure variable as a continuous measure did not change the overall results.

\section{Conclusion}

Even after adjusting for sociodemographic factors, people with diabetes who find dimensions of health literacy difficult have higher odds of being physically inactive and having unhealthy dietary habits compared to people who do not have these difficulties. Strategies for improving physical activity and diet among people with diabetes may benefit by having focus on health literacy within prevention, patient education, and other public health interventions.

\section{Competing Interests}

The authors declare that there are no competing interests regarding the publication of this paper.

\section{Acknowledgments}

This study was funded by the Central Denmark Region. Rebecca K. Simmons is supported by the Danish Diabetes Academy, which is funded by the Novo Nordisk Foundation. Helle Terkildsen Maindal works at the Steno Health Promotion Centre funded by Novo Nordisk A/S and the Novo Nordisk Foundation. The authors do not perceive this as a conflict of interests in this study.

\section{References}

[1] International Diabetes Federation, IDF Diabetes Atlas 2015, 7th edition, 2015.

[2] P. Gæde, P. Vedel, N. Larsen, G. V. H. Jensen, H.-H. Parving, and O. Pedersen, "Multifactorial intervention and cardiovascular disease in patients with type 2 diabetes," The New England Journal of Medicine, vol. 348, no. 5, pp. 383-393, 2003.

[3] L. Ryden, E. Standl, M. Bartnik et al., "Guidelines on diabetes, pre-diabetes, and cardiovascular diseases: executive summary. The Task Force on Diabetes and Cardiovascular Diseases of the European Society of Cardiology (ESC) and of the European Association for the Study of Diabetes (EASD)," European Heart Journal, vol. 28, no. 1, pp. 88-136, 2007.

[4] D. Nutbeam, "Health promotion glossary," Health Promotion, vol. 1, no. 1, pp. 113-127, 1986.

[5] K. Friis, M. Lasgaard, R. H. Osborne, and H. T. Maindal, "Gaps in understanding health and engagement with healthcare providers across common long-term conditions: a population survey of health literacy in 29473 Danish citizens," BMJ Open, vol. 6, no. 1, Article ID e009627, 2016.

[6] F. Al Sayah, S. R. Majumdar, B. Williams, S. Robertson, and J. A. Johnson, "Health literacy and health outcomes in diabetes: a systematic review," Journal of General Internal Medicine, vol. 28, no. 3, pp. 444-452, 2013.

[7] S. C. Bailey, A. G. Brega, T. M. Crutchfield et al., "Update on health literacy and diabetes," Diabetes Educator, vol. 40, no. 5, pp. 581-604, 2014.

[8] J. G. Souza, D. Apolinario, R. M. Magaldi, A. L. Busse, F. Campora, and W. Jacob-Filho, "Functional health literacy and glycaemic control in older adults with type 2 diabetes: a crosssectional study," BMJ Open, vol. 4, no. 2, Article ID e004180, 2014.

[9] D. Schillinger, K. Grumbach, J. Piette et al., "Association of health literacy with diabetes outcomes," The Journal of the American Medical Association, vol. 288, no. 4, pp. 475-482, 2002.

[10] R. J. Adams, C. Piantadosi, K. Ettridge et al., "Functional health literacy mediates the relationship between socio-economic 
status, perceptions and lifestyle behaviors related to cancer risk in an Australian population," Patient Education and Counseling, vol. 91, no. 2, pp. 206-212, 2013.

[11] B. Geboers, A. F. de Winter, K. A. Luten, C. J. M. Jansen, and S. A. Reijneveld, "The association of health literacy with physical activity and nutritional behavior in older adults, and its social cognitive mediators," Journal of Health Communication, vol. 19, supplement 2, pp. 61-76, 2014.

[12] O. Husson, F. Mols, M. P. Fransen, L. V. Van De Poll-Franse, and N. P. M. Ezendam, "Low subjective health literacy is associated with adverse health behaviors and worse health-related quality of life among colorectal cancer survivors: results from the profiles registry," Psycho-Oncology, vol. 24, no. 4, pp. 478-486, 2015.

[13] K. E. Speirs, L. A. Messina, A. L. Munger, and S. K. Grutzmacher, "Health literacy and nutrition behaviors among low-income adults," Journal of Health Care for the Poor and Underserved, vol. 23, no. 3, pp. 1082-1091, 2012.

[14] C. von Wagner, K. Knight, A. Steptoe, and J. Wardle, "Functional health literacy and health-promoting behaviour in a national sample of British adults," Journal of Epidemiology and Community Health, vol. 61, no. 12, pp. 1086-1090, 2007.

[15] M. S. Wolf, J. A. Gazmararian, and D. W. Baker, "Health literacy and health risk behaviors among older adults," American Journal of Preventive Medicine, vol. 32, no. 1, pp. 19-24, 2007.

[16] S. S. Bains and L. E. Egede, "Associations between health literacy, diabetes knowledge, self-care behaviors, and glycemic control in a low income population with type 2 diabetes," Diabetes Technology and Therapeutics, vol. 13, no. 3, pp. 335-341, 2011.

[17] S. Kim, F. Love, D. A. Quistberg, and J. A. Shea, "Association of health literacy with self-management behavior in patients with diabetes," Diabetes Care, vol. 27, no. 12, pp. 2980-2982, 2004.

[18] V. McCleary-Jones, "Health literacy and its association with diabetes knowledge, self-efficacy and disease self-management among African Americans with diabetes mellitus," The ABNF Journal, vol. 22, no. 2, pp. 25-32, 2011.

[19] F. Al Sayah, S. R. Majumdar, L. E. Egede, and J. A. Johnson, "Associations between health literacy and health outcomes in a predominantly low-income African American population with type 2 diabetes," Journal of Health Communication, vol. 20, no. 5, pp. 581-588, 2015.

[20] E. Eyüboğlu and P. J. Schulz, "Do health literacy and patient empowerment affect self-care behaviour? A survey study among Turkish patients with diabetes," BMJ Open, vol. 6, no. 3, Article ID e010186, 2016.

[21] A. I. Christensen, M. Davidsen, O. Ekholm, P. V. Pedersen, and K. Juel, The Health of the Danes-The National Public Health Survey 2013, 2014.

[22] R. H. Osborne, R. W. Batterham, G. R. Elsworth, M. Hawkins, and R. Buchbinder, "The grounded psychometric development and initial validation of the Health Literacy Questionnaire (HLQ)," BMC Public Health, vol. 13, article 658, 2013.

[23] A. Bo, K. Friis, R. H. Osborne, and H. T. Maindal, "National indicators of health literacy: ability to understand health information and to engage actively with healthcare providers-a population-based survey among Danish adults," BMC Public Health, vol. 14, article 1095, 2014.

[24] U. Toft, L. H. Kristoffersen, C. Lau, K. Borch-Johnsen, and T. Jørgensen, "The Dietary Quality Score: validation and association with cardiovascular risk factors: the Inter99 study," European Journal of Clinical Nutrition, vol. 61, no. 2, pp. 270-278, 2007.
[25] Kræftens Bekæmpelse, Rygeloven-Lovtekster [Smoking BansWording of the Act], 2013, https://www.cancer.dk/forebyg/rensluften/rygeloven/lovtekster/.

[26] H. Ishikawa, T. Takeuchi, and E. Yano, "Measuring functional, communicative, and critical health literacy among diabetic patients," Diabetes Care, vol. 31, no. 5, pp. 874-879, 2008.

[27] K. M. Robinson, M. Lykke, B. H. Hansen et al., Health Profile for the Region and Municipalities, Research Centre for Prevention and Health, Capital Region of Denmark, 2014.

[28] T. C. Davis, S. W. Long, R. H. Jackson et al., "Rapid estimate of adult literacy in medicine: a shortened screening instrument," Family Medicine, vol. 25, no. 6, pp. 391-395, 1993.

[29] R. M. Parker, D. W. Baker, M. V. Williams, and J. R. Nurss, "The test of functional health literacy in adults: a new instrument for measuring patients' literacy skills," Journal of General Internal Medicine, vol. 10, no. 10, pp. 537-541, 1995.

[30] B. D. Weiss, M. Z. Mays, W. Martz et al., "Quick assessment of literacy in primary care: the newest vital sign," Annals of Family Medicine, vol. 3, no. 6, pp. 514-522, 2005.

[31] R. Buchbinder, R. Batterham, G. Elsworth, C. E. Dionne, E. Irvin, and R. H. Osborne, "A validity-driven approach to the understanding of the personal and societal burden of low back pain: development of a conceptual and measurement model," Arthritis Research \& Therapy, vol. 13, no. 5, article R152, 2011. 


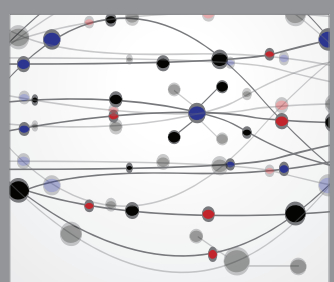

The Scientific World Journal
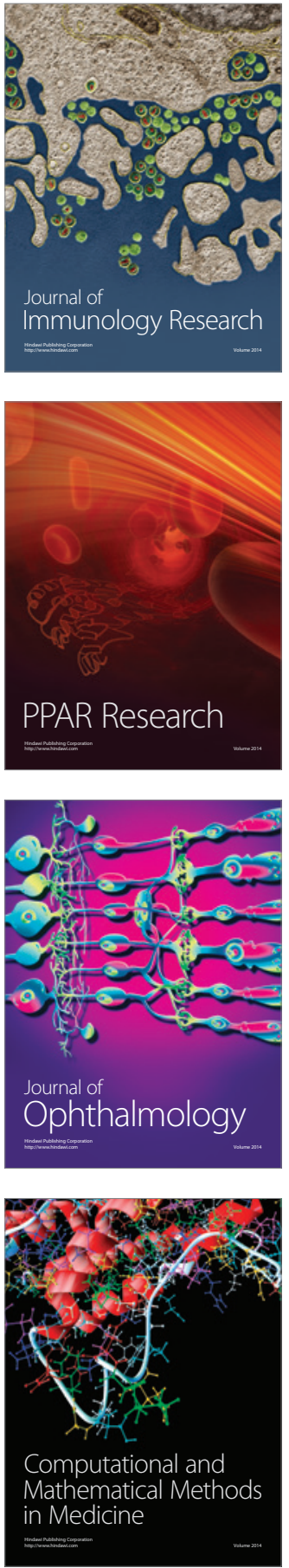

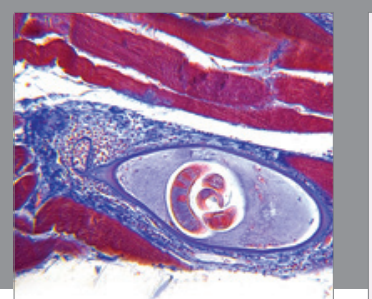

Gastroenterology Research and Practice

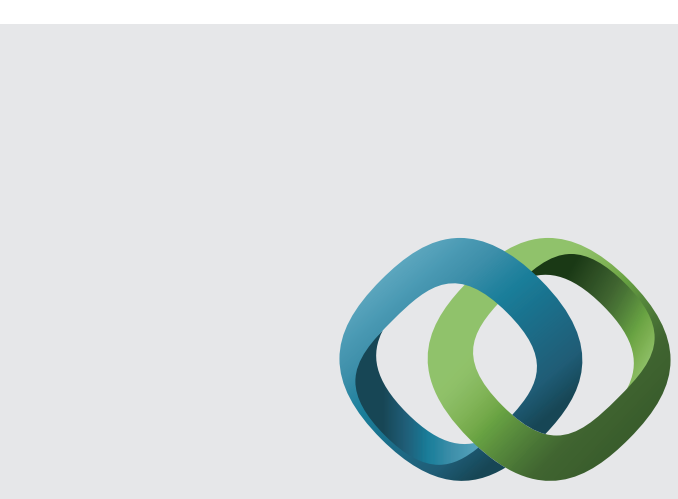

\section{Hindawi}

Submit your manuscripts at

http://www.hindawi.com
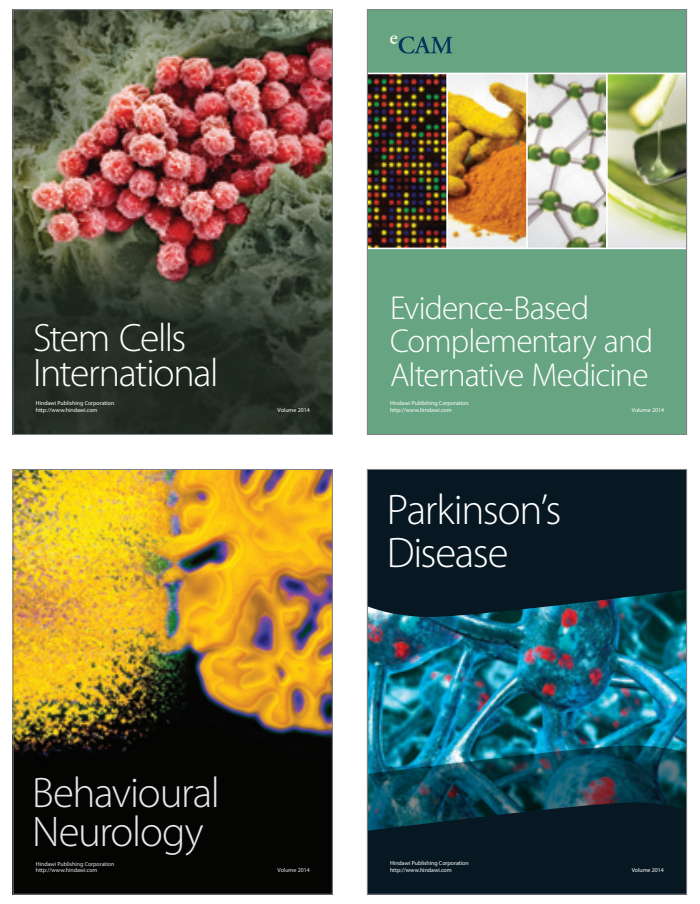
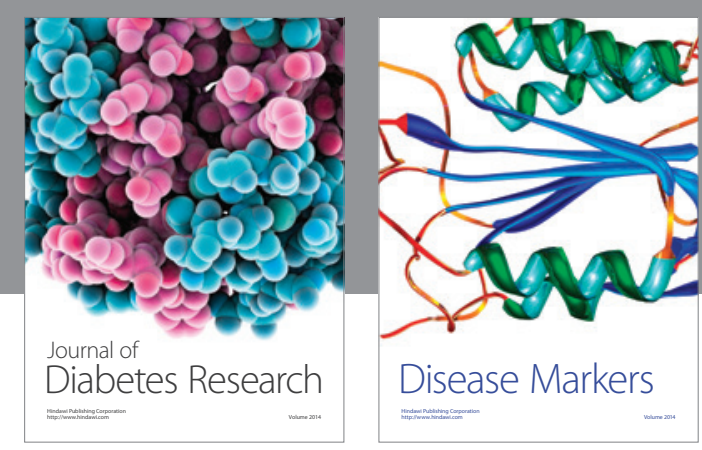

Disease Markers
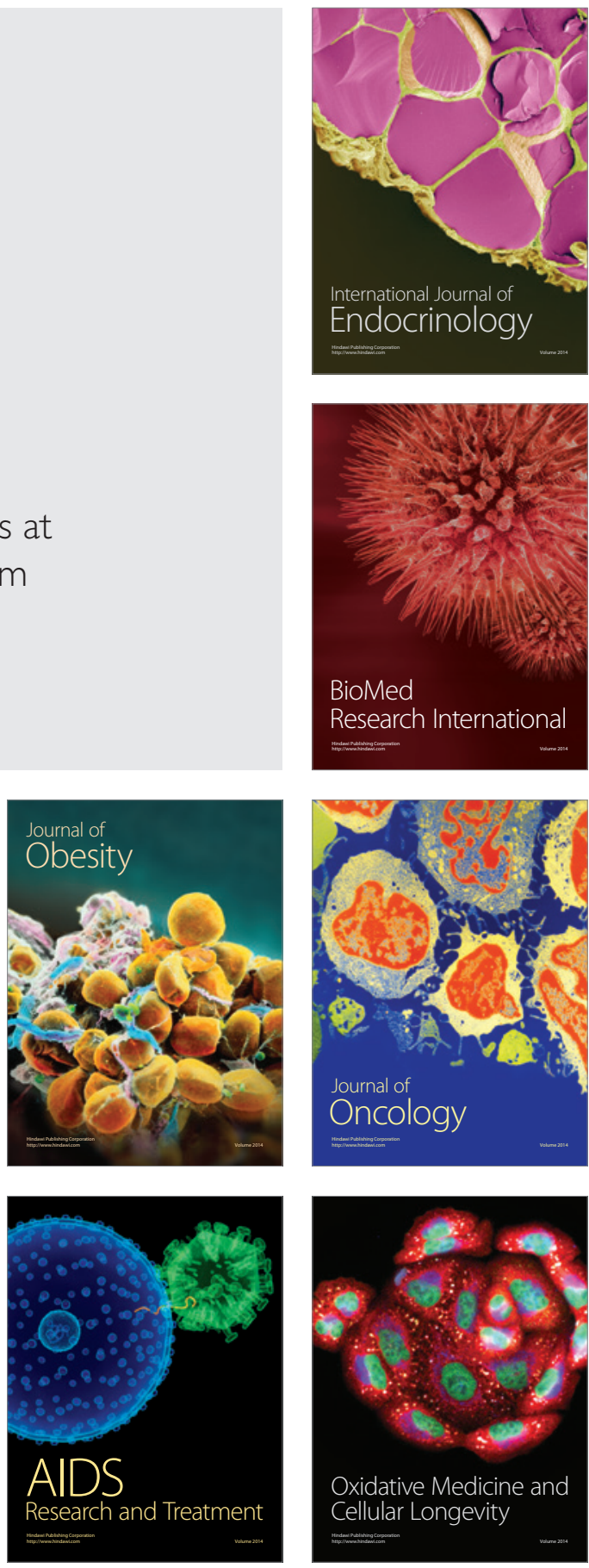